\title{
Autonomous Golf Ball Picking Robot Design and Development
}

\author{
NINO PEREIRA ${ }^{1}$, FERNANDO RIBEIRO $^{1}$, GIL LOPES $^{1}$, DANIEL WHITNEY $^{2}$, \\ JORGE LINO 3 \\ ${ }^{1}$ University of Minho, Campus de Azurém, 4800-058 Guimarães, Portugal, martins@sarobotica.pt, \\ fernando@dei.uminho.pt,gil@dei.uminho.pt \\ ${ }^{2}$ MIT, Cambridge,MA,02139,USA,dwhitney@mit.edu \\ ${ }^{3}$ FEUP, Rua Dr. Roberto Frias, 4200-465 Porto,Portugal,falves@fe.up.pt
}

\begin{abstract}
This paper presents the methodology and the results on the design and development of an autonomous golf ball picking robot. The strategy followed to develop a commercial product is presented, based on prior identification requirements, which consists of picking up golf balls on a Driving Range in a safe and efficient way. A functional prototype was built and tested. It has autonomy of up to $8 \mathrm{~h}$ per day. It is capable of collecting up to 1200 balls in one single journey. It weighs $130 \mathrm{~kg}$ and is capable to climb slopes of up to $22^{\circ}$. The maximum speed is $8 \mathrm{~km} / \mathrm{h}$ and the robot takes $140 \mathrm{~min}$ to completely sweep a 25,000 m2 field at 7.2 $\mathrm{km} / \mathrm{h}(2 \mathrm{~m} / \mathrm{s})$ average speed.

A hybrid information system was implemented in order to provide a statistically relevant prediction of golf balls location. Autonomous navigation was developed and tested on a simulation environment. Preliminary results showed that the new path planning algorithm Twin-RRT* is able to form closed loop trajectories and improve the result over time. Kinematic constraints were already taken into account on the algorithm. This sampling based algorithm has potential usage in solving other TPP (Travelling Purchaser Problem) related problems.
\end{abstract}

Two patents are pending concerning the technological novelties of this work.

Keywords: autonomous mobile field robotics, golf ball picking robot, differential drive kinematics, path planning

\section{INTRODUCTION}

In 1996 at the International Conference on Robotics and Automation (ICRA), several panelists stated that the academic research going out on their labs often does not produce visible results until later. Fundamental research is quite distant to practical and useful day-to-day applications. The industrial or commercial sector rarely benefits from the academic research (Pack et al., 1997). Although many researchers have shifted their focus to better suit people needs and solve practical problems, there are still few examples of success achieved in short periods of time. 
The project here presented is focused to deliver a commercial product in a very narrow time frame. Researchers at the Automation and Robotics Laboratory of University of Minho have been developing autonomous robots since 1998 (Machado et al., 1999b). Focusing on vision systems (Machado et al., 1999a, Lima et al., 2001) and different mobile platforms (Ribeiro et al., 2004, Pacheco, 2008, Ribeiro, 2007b), the group has contributed to improve people's life by the use of autonomous or semi-autonomous mobile platforms (Ribeiro, 2007a).

The idea of developing an autonomous mobile robot which could collect golf balls came from the fact that Driving Ranges accumulate over 10,000 balls per day and requires an intensive and tedious human labor even when accomplished with the help of motorized devices (Pacheco, 2008). To perform such task a successful device would have to be able to maintain clear a $25,000 \mathrm{~m}^{2}$ field, collecting about 10,000 balls per day, avoiding obstacles and climbing slopes up to 20 degrees. It should operate autonomously and be resistant to corrosion since it would be navigating on external environment. Additional to these requirements the robot should also be efficient, maximizing the number of balls collected over the travelled distance. This paper describes the research and development work carried out to develop a commercial robot that responds to this ambitious goal.

First, a detailed problem analysis and product development strategy is presented. The list of requirements and technical specifications is established. The following section introduces the final concept that meets the identified requirements and describes the methods to validate the hardware and functional behavior. Afterwards, autonomous navigation simulations results and field test performance evaluation of the industrial prototype are described. Finally, the main conclusions and further development directions are presented.

\section{PROBLEM ANALYSIS AND PRODUCT DEVELOPMENT}

A set of milestones (Fig. 1Fig. 1) was established following the ideas of the product development process proposed by Ulrich and Eppinger (Ulrich and Eppinger, 2004). The project was subdivided into two main phases. Phase I comprises the correct identification of the need, the specifications of technical requirements, the concept generations and the final concept selection. Phase II involves structural (hardware) and functional validation, prototyping and field tests as depicted in Fig. 1. 


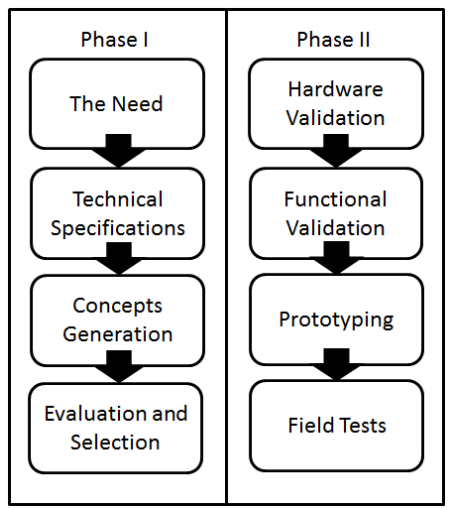

Fig. 1 - Project Milestones

\subsection{The Need}

In golf practice fields, the main requirement consists of always guaranteeing balls availability for players. This need leads to the next one, which is preventing the ball dispensing machine from being empty, and consequently there is the need for some method, human or mechanical, to constantly replenish the machine by picking up the golf balls and put them back into the dispensing machine (Fig.

Fig. 2).

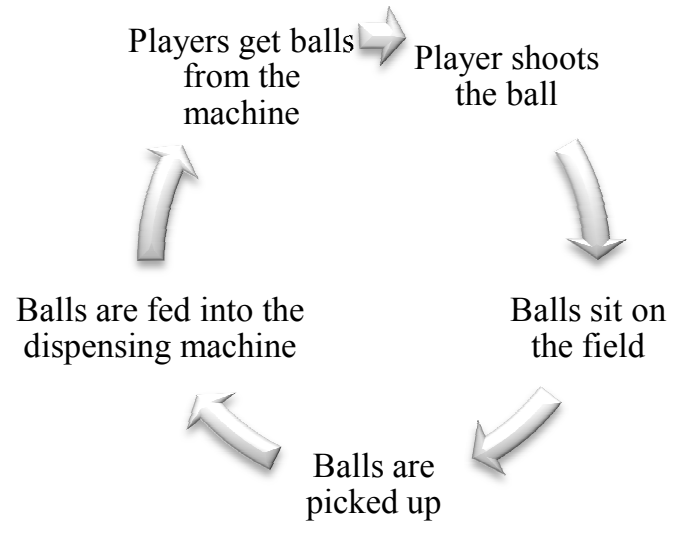

Fig. 2 - Golf ball cycle on a golf practice field

\subsection{State of the Art}

\subsubsection{Traditional means}

The golf ball picking task is, in many cases, performed by a worker, carrying just a basket (Pacheco, 2008). Such work is considered to be tedious and causes many health problems related to back pain (De Looze et al., 1995).

Even when it is performed with the help of mechanical devices, such as carts, or trolleys, which may 
improve the efficiency, they also require a big effort and still produce significant fatigue.

\subsubsection{Buggy Solutions}

The buggy solution is adopted as a more effective way to collect the golf balls. Relatively fast collecting rates are achieved (4,000 balls/hour) and high capacity baskets (1,800 balls) are used. Nevertheless, they are also human operated and require a significant initial investment. This is a problem because on one hand, requires a worker to operate the machine which has a higher cost on human labor, and on the other hand, for low usage rates, it is significantly more expensive taking into account the maintenance cost/operation time ratio. Furthermore, the buggy needs parking space which has an additional cost.

\subsubsection{Autonomous and Semi-autonomous devices}

Researchers are already engaged on finding new approaches that could improve the golf ball picking task (Ming-Shyan Wang, 2006, Pacheco, 2008, Sun-Li et al., 2005). Some use vision systems to find and pursuit the golf balls (Sun-Li et al., 2005, Pacheco, 2008). Others use hand based movements to drive robots on the field (Ming-Shyan Wang, 2006). As pointed out before, the need for dedicated and specialized vehicles to pick up golf balls is becoming a must (Ming-Shyan Wang, 2006) to reduce overall costs and to decrease the risk and health problems for humans.

There is an autonomous robot available in the market which aims to do this task in a simple manner (Automation, 2011). It moves randomly within a wire limited perimeter and collects all the balls that appear on its way. This robot has a 400 balls capacity container, weighs about $65 \mathrm{~kg}$ and operates at speeds up to 3.6 $\mathrm{km} / \mathrm{hour}$.

Vision systems often require an exhaustive and delicate calibration process (Yang et al., 2009, Li and Chen, 2003), however, on external environments, this can be a major problem. Light variations, rain and dirt can severely affect the vision systems (Jinwei et al., 2009). Direct human commands such as gestures, voice commands, keyboard or touch screen inputs are all important machine interfaces which have been widely used. Nevertheless, being autonomous, means to be able to operate without direct orders at all times (Wada, 2009).

\subsection{Technical Specifications}

A panel of 12 experts assisted the establishment of the requirements list. The developed solution considered some constraints which were established following the feedback from the Portuguese Golf Federation, including golf associations and golf course managers in a total of 12 persons. The main constraints were: final price lower than $12,000 €$; collecting rate of about 10,000 balls/day; robot cover material resistant to ball impact, oxidation and corrosion.

To meet the initial constraints, a list of target technical specifications was established and ranked according to its relative importance (Table I). The metric unit and target value is shown where applicable. Some of these metrics are subjective and should be later evaluated by users or observation. 
TABLE I

\begin{tabular}{cc} 
LiST OF RANKED TECHNICAL SPECIFICATIONS & \\
\hline \hline Metric & Unit \\
\hline Intuitive and simple usage & Subjective \\
Autonomy & $8 \mathrm{~h} /$ day \\
Container Capacity & $1,200 \mathrm{balls}$ \\
Manually unlock the robot & Yes \\
Humidity, oxidation and corrosion resistance & Subj. \\
Remote operation & Yes \\
Maintenance capacity & $25,000 \mathrm{~m}{ }^{2}$ \\
Minimum turning angle & $15^{\circ}$ \\
Collecting rate & $1,500 \mathrm{balls} / \mathrm{h}$ \\
Minimum wheel diameter & $20 \mathrm{~cm}$ \\
Maximum weight & $100 \mathrm{~kg}$ \\
Total field periodic scanning & once $/ \mathrm{week}$ \\
Publicity area & $1 \mathrm{~m}{ }^{2}$ \\
Target Speed & $1,4 \mathrm{~m} / \mathrm{s}$ \\
Maximum slope & $15^{\circ}$ \\
Maximum height & $60 \mathrm{~cm}$ \\
Trouble diagnostics & Yes \\
Self-localization & $\mathrm{GPS}$ \\
Ball counting & Yes \\
Width & $1,5 \mathrm{~m}$ \\
Number of disks & 30 \\
\hline \hline
\end{tabular}

These target specifications will be re-evaluated all along the entire development process. The implemented features were established following these specifications as guidelines. A set of 18 initial concepts was produced from which a final concept evolved (described in the next section). The process of screening and selection will not be described because it is outside the scope of this paper.

\section{FINAL CONCEPT AND PROTOTYPE}

\subsection{System Architecture}

The system comprises the autonomous moving vehicle (robot), a monitoring station (optional), and a remote control, which can be used to override the autonomous control.

The mechanical chassis (Fig. 3) was designed to accomplish two DC motors directly coupled to the two rear wheels. The robot movement is controlled by electric differential. This type of coupling reduces the mechanical losses. The free wheel at the robot front prevents it from getting stuck when facing a higher slope. 


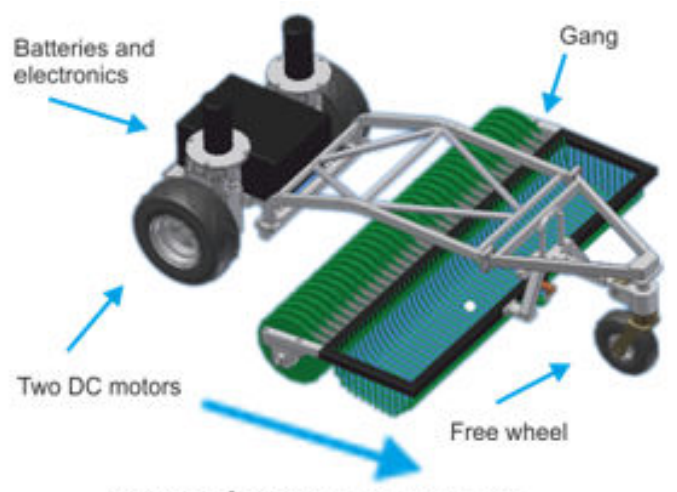

Motion direction in operation

Fig. 3 - Robot mechanical chassis (apparatus)
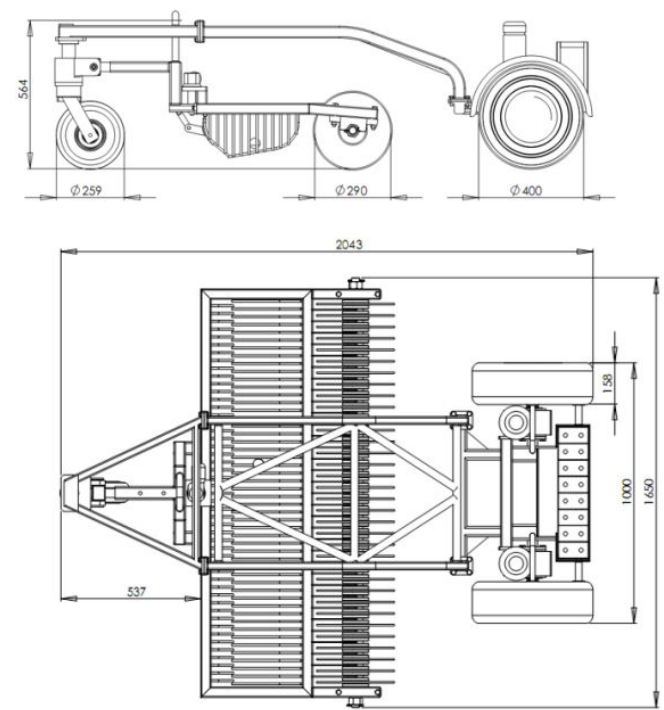

Fig. 4 - Technical Drawing

The robot structure (Fig. 4) was made compatible with currently commercially available gangs (set of disks that pick up the golf balls) allowing golf practice fields a soft migration from existing buggy systems to an autonomous solution without extra costs in gangs. A set of 34 polyethylene disks with a diameter of 29.4 $\mathrm{cm}$ each, are mounted on a steel axle. The gang is attached to the front structure and has three degrees of freedom ( $40^{\circ}$ Roll, $10^{\circ}$ Pitch and $20^{\circ}$ Yaw amplitudes). The Yaw amplitude was limited to $20^{\circ}$ in order to prevent damage to the gang disks. The balls are recovered by the gang disks when they roll over the balls. Since the disks rotate as the robot moves, the balls get stuck in between the disks and travel up as the disks rotate. When the balls reach the top of the basket a wedge forces them to detach from the disks and they drop into the basket. The maximum turning angle of the gang is limited to about 30 degrees (horizontal). The maximum width is $1.65 \mathrm{~m}$. The whole structure (mechanical chassis, motors, batteries, wheels and gang) weighs $130 \mathrm{~kg}$ and it is prepared to pull up to $200 \mathrm{~kg}$. Its capacity is about 1200 balls which means $54 \mathrm{~kg}$ 
extra. The chassis is over the original estimated maximum weight $(100 \mathrm{~kg})$ but the structure will be lighter on its final version.

The monitoring station is an optional item. It provides information in real time about robot's activity, with a friendly interface to send commands to the robot and to receive feedback information. Some parameters configuration might be required at the installation procedure. It can be used to view or change some configurations and eventually adjust some parameters in real time operation.

The remote control provides manual control of the robot, should it be required. When started, the manual operation gives the user full control of the robot movement. Besides that, the remote control has bidirectional communication using the Zigbee protocol. This means that the robot may send alerts to this device, notifying the operator when necessary.

The robot is equipped with sensors to provide the necessary information for moving the robot safely and efficiently. These include a gyroscope, temperature and humidity sensors, encoders coupled to the motors, sonars, GPS, compass, touch sensors, voltage and current sensors.

A ball counter device using micro-switches provides information about the number of balls the robot collects. Sonars are used to detect obstacles so they can be avoided autonomously. The differential GPS system informs the robot about its location on the field.

Other functions and devices are identified and summarized on Fig. 5.

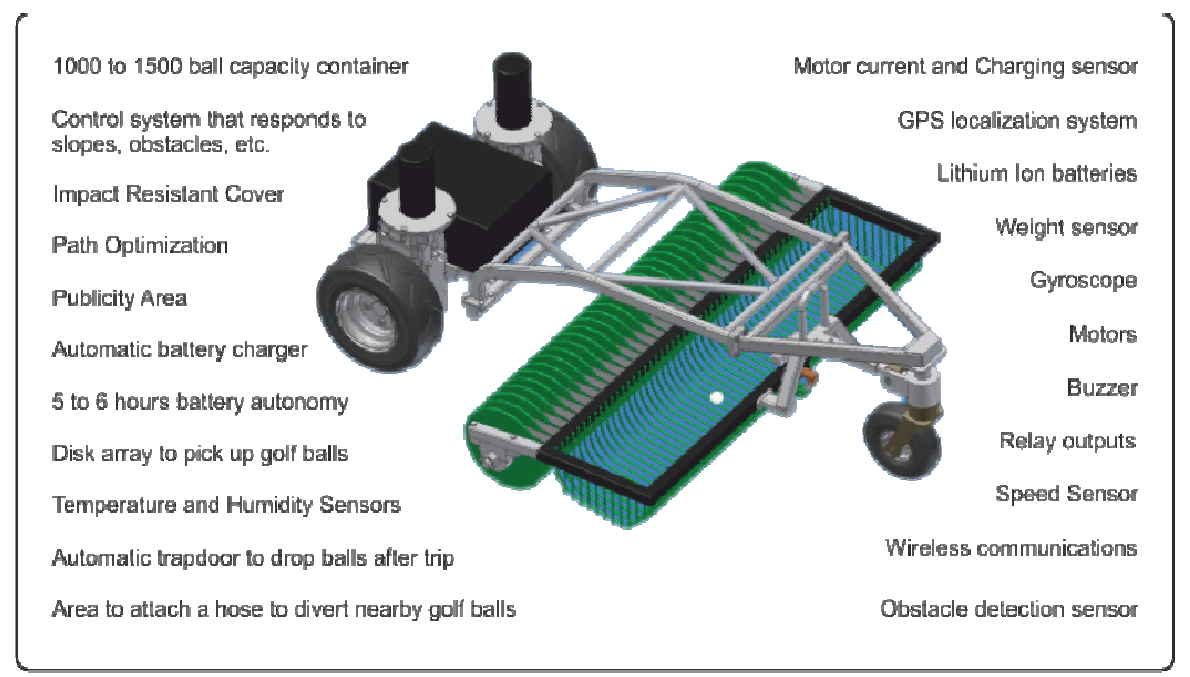

Fig. 5 - Robot system overview - functions and devices

Two 24V DC motors, each one attached to one wheel, are used to control the robot movement. An electrical differential is used to control robot heading direction. These DC motors have permanent magnets with electric brake and 1:30 gearbox incorporated. Each motor consumes $800 \mathrm{~W}$ and has a gearbox output of $100 \mathrm{rpm}$. Using $400 \mathrm{~mm}$ diameter wheels, the maximum linear speed is $7.5 \mathrm{~km} / \mathrm{h}$.

Eight cells of $3.6 \mathrm{~V}, 160$ Ah lithium ion batteries are used to power the robot. With these specifications, 
the robot operates for about 8 hours, after which it needs a 3 hours recharge. Nominal current is $80 \mathrm{~A}$, and maximum current is $480 \mathrm{~A}$.

A controller for three motors was developed (Fig. 6) to meet the system requirements in terms of power and current. It is an $\mathrm{I}^{2} \mathrm{C}$ slave device that receives speed and direction instructions and controls the motors accordingly, using feedback information from encoders. The motor controller features are summarized on Table 2 .

TABLE 2

LIST OF MOTOR CONTROLLER FEATURES

\begin{tabular}{cc}
\hline \hline Main Features & \\
\hline Nominal Current / motor & $8 \mathrm{~A}$ \\
Maximum Current / motor & $120 \mathrm{~A}$ \\
Maximum Input Voltage & $36 \mathrm{~V}$ \\
Nominal Input Voltage & $24 \mathrm{~V}$ \\
Cut-off Temperature & $100^{\circ} \mathrm{C}$ \\
Communication Bus & $\mathrm{I}^{2} \mathrm{C}$ \\
\hline \hline
\end{tabular}

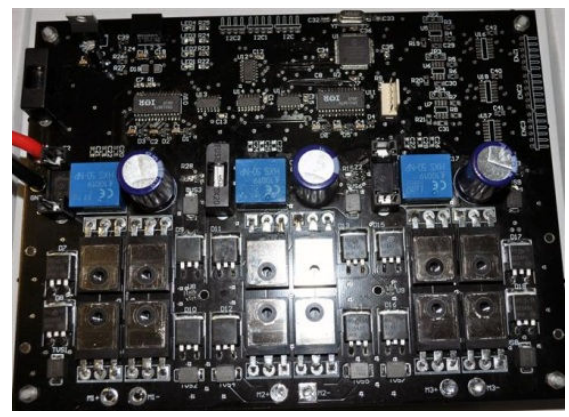

Fig. 6 - OMNI_3MD_MAX - 3 motors driver board

\subsection{Kinematic Analysis}

The Golfminho Robot drive mechanism is a differential drive which results from a combination of simple differential drive and an Ackerman car model (Dudek and Jenkin, 2010). It differs from the tricycle mechanism because the traction wheels are on the rear. Both wheels are mounted on a common axis and each one may be driven either forward or backward at different speeds. The front wheel does not limit the movement but the gang does. 


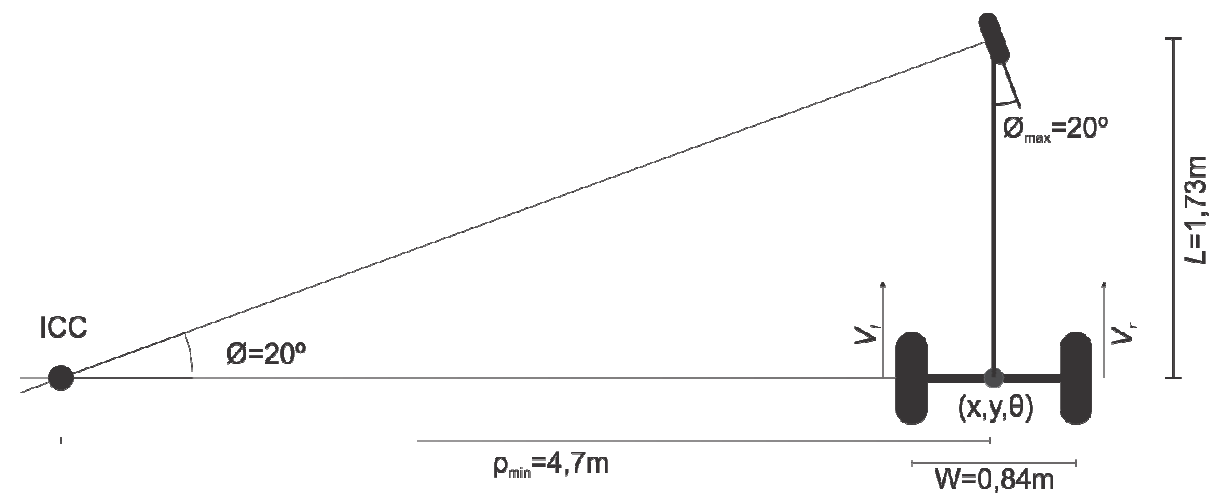

Fig. 7 - Left turn differential drive representation

The maximum Yaw angle is limited to about $20^{\circ}$. This value is used to calculate for the minimum curvature radius $\left(\rho_{\min }\right)$.

$$
\rho_{\min }=\frac{L}{\tan (\varnothing)}=\frac{1,73 \mathrm{~m}}{\tan \left(20^{\circ}\right)}=4,7 \mathrm{~m}
$$

Note: $\varnothing=$ Gang Maximum Yaw Angle $=20^{\circ}$

Varying the velocity of each wheel, the robot may turn right or left, rotating about a pivot point known as ICC - Instantaneous Center of Curvature, lying along their common left and right wheel axis. The ICC point is determined by the intersection point of the line along the axis of the front wheel and the line along the axis of the rear wheels (Fig. 107 ).

The angular velocity, $\omega$, and the curvature radius, $\rho$, may be calculated solving the pair of equations:

$$
\text { left turn: }\left\{\begin{array} { l } 
{ \omega ( \rho + \frac { W } { 2 } ) = V _ { r } } \\
{ \omega ( \rho - \frac { W } { 2 } ) = V _ { l } }
\end{array} \text { or right turn: } \quad \left\{\begin{array}{l}
\omega\left(\rho-\frac{W}{2}\right)=V_{r} \\
\omega\left(\rho+\frac{W}{2}\right)=V_{l}
\end{array}\right.\right.
$$

Solving for $\omega$ and $\rho$, we obtain the expressions:

$$
\rho=\frac{W}{2} \times \frac{V_{r}+V_{l}}{V_{r}-V_{l}} \quad \omega=\frac{V_{r}-V_{l}}{W}
$$

Also the relation between $V_{r}$ and $V_{1}$ for any case may be calculated. For the case of Golfminho Robot, this means that when turning left at maximum angle $\left(\varnothing=20^{\circ}\right), \frac{V_{l}}{V_{r}}=83,5 \%$.

It is possible to determine the robot next pose $\left(x^{\prime}, y^{\prime}, \theta^{\prime}\right)$, knowing the velocity of each wheel $\left(V_{l}\right.$ and $\left.V_{r}\right)$, the current pose $(x, y, \theta)$ and the elapsed time $\delta t$. 
The following general equations apply for this type of differential robot (Choset, 2005, Dudek and Jenkin, 2010, Latombe, 1991).

\section{Left Turn:}

$$
I C C=[x-\rho \sin (\theta), y+\rho \cos (\theta)]
$$

At $\mathrm{t}+\delta \mathrm{t}$ :

$$
\left[\begin{array}{l}
x^{\prime} \\
y^{\prime} \\
\theta^{\prime}
\end{array}\right]=\left[\begin{array}{ccc}
\cos (\omega \delta t) & -\sin (\omega \delta t) & 0 \\
\sin (\omega \delta t) & \cos (\omega \delta t) & 0 \\
0 & 0 & 1
\end{array}\right] \times\left[\begin{array}{c}
x-I C C x \\
y-I C C y \\
\theta
\end{array}\right]+\left[\begin{array}{c}
I C C x \\
I C C y \\
\omega \delta t
\end{array}\right]
$$

\section{Right Turn:}

$$
\text { ICC }=[x+\rho \sin (\theta), \quad y-\rho \cos (\theta)]
$$

At $\mathrm{t}+\delta \mathrm{t}$ :

$$
\left[\begin{array}{l}
x^{\prime} \\
y^{\prime} \\
\theta^{\prime}
\end{array}\right]=\left[\begin{array}{ccc}
\cos (\omega \delta t) & \sin (\omega \delta t) & 0 \\
-\sin (\omega \delta t) & \cos (\omega \delta t) & 0 \\
0 & 0 & 1
\end{array}\right] \times\left[\begin{array}{c}
x-I C C x \\
y-I C C y \\
\theta
\end{array}\right]+\left[\begin{array}{l}
I C C x \\
I C C y \\
-\omega \delta t
\end{array}\right]
$$

\subsection{Structural and Functional Validation}

The main hardware platform was subject to testing on a simulation platform. The Webots ${ }^{\mathrm{TM}} 6$ (http://www.cyberbotics.com) simulation tool was used to accomplish two objectives: to establish a ground truth model of the world and to validate the robot hardware operation and behavior.

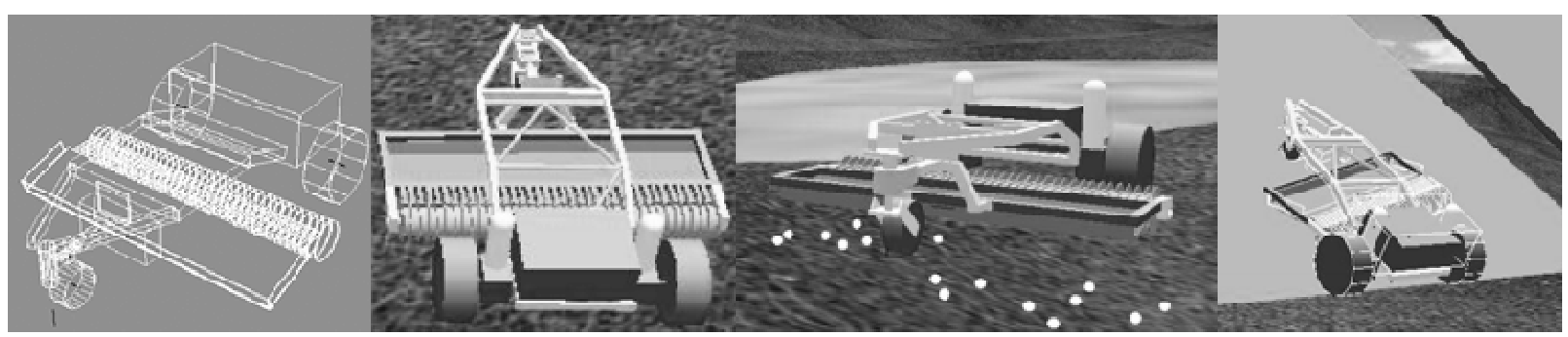

Fig. 8 - Platform structural design and functional validation using Webots ${ }^{\mathrm{TM}}$ simulation tool

It is important to point out that all parts and joints were carefully designed respecting dimensions, toughness, density and roughness of the real parts and materials.

Four main tests were performed using this tool: motion control, picking performance, ramp test and free run on an irregular terrain (Fig. 8).

The most important conclusions driven from these tests showed that the robot is not expected to overcome slopes higher than $30^{\circ}$, the motion controller should take into account the hardware limitations such as the gang maximum yaw angle so to preserve the integrity of the disks and the robot speed does not affect the picking ability which was $100 \%$ effective in the simulation tests. 


\subsection{Autonomous Navigation}

Every planning strategy relies on knowing the exact location of the targets, in order to optimize the task, reducing the pickup time, the energy required or any other functional cost.

A real important issue to solve is the fact that the golf balls location is unknown. So, the first problem to deal with was the lack of detailed information about the targets position. A "Hybrid Localization System" was proposed which relies on statistical information and it uses both real and virtual data to predict the actual balls location on the field.

\subsubsection{Hybrid Localization System}

The hybrid localization system provides a virtual map containing previewed golf ball location, which is continuously being updated, based on real information gathered by the robot. The system logs relevant data, such as date, time and GPS data for each collected ball. The data is then processed to retrieve statistically relevant information, such as the distribution probability, the frequency at which balls are sent to the field and respective variations along the day, week, month and year. A virtual map is generated and continuously updated.

Fig. 9 shows a diagram of the operating system. It includes a Real Item Database, a Trigger Unit, a Virtual Item Generator, a Virtual Item Positioner a Virtual Map and an Area Clearance module. The Trigger Unit is a software module which generates a signal to start an event. The signal may have a fixed or variable frequency. This frequency is continuously adjusted based on the Real Item Database information. The Virtual Item Generator is another software module which generates a virtual item (golf ball) whenever it receives the trigger signal from the Trigger Unit. The virtual item generated has the same characteristics (which can be detected or input to the system) of a real one.

The Virtual Item Positioner is responsible for allocating the item generated by the Virtual Item Generator in the Virtual Map. It assigns a position to the virtual item on the Virtual Map.

The Virtual Map retains an updated and statistically significant prediction of the golf balls on the field. It constitutes the most important output of the system.

The Area Clearance is a module that acts on the Virtual Map removing items from a specific or entire area of the field. While navigating on the field, the robot sweeps areas that will be cleared from the Virtual Map, i.e. whether or not it will pick balls in the reality, virtually it will collect all the balls in that same area. This last effect could be interpreted as an augmented virtuality, in the same way as one conceived an augmented reality. 


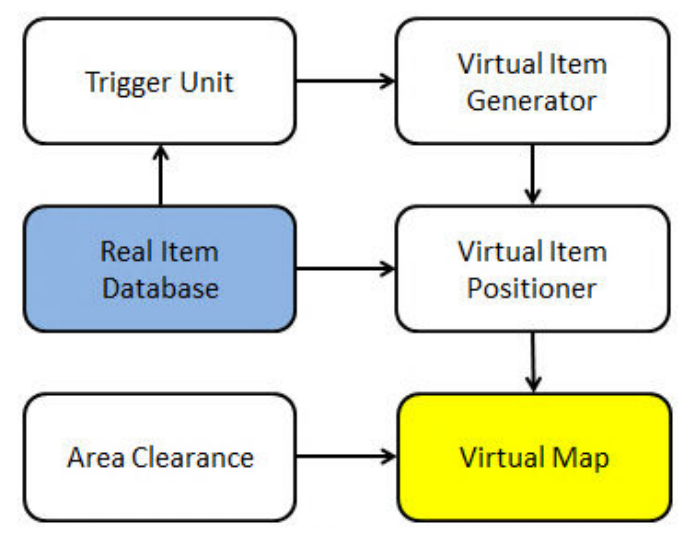

Fig. 9 - Hybrid Location System Diagram

Fig. 10 represents an example of the Real and the Virtual Field. The Virtual Field is expected to have approximately the same number of items present on the Real Field. The error will be smaller for larger number of items. The distribution of the predicted items will be close to the real distribution. The discrepancy between the Virtual and Real position will decrease as the Real Database increases in size and the total number of items increase.
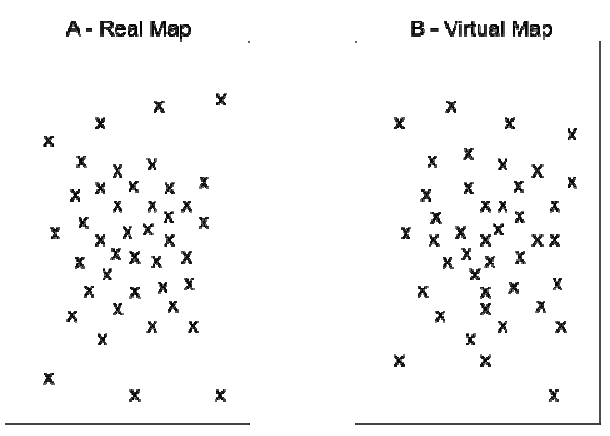

Fig. 10 - Real and Virtual Field Representations

It is expected that the robot performance will continuously be improved as it operates repeatedly.

Guessing the golf balls location is only part of the solution. Another, and perhaps even more challenging problem is to determine the most efficient pathway to collect the maximum amount of balls over a determined distance travelled or time. Typically a path planning problem deals with a starting position and one target position. In this case there is also a starting and ending position with many possible targets, but none of them is compulsory (Fig. 11). This problem shares many similarities with the TPP (Traveling Purchaser Problem). It is a NP-hard problem studied in theoretical computer science. TPP was first described by Ramesh (Ramesh, 1981) in 1981. He also proposed an exact algorithm which solves the problem optimally. However, TPP solutions have always been computationally very expensive (Pearn and Chien, 1998). Since then some attempts have been made to deal with that complexity (Golden et al., 1981, Ong, 1982, Ravi and Salman, 1999, Singh and van Oudheusden, 1997). 
a)

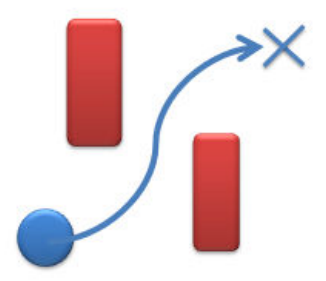

b)

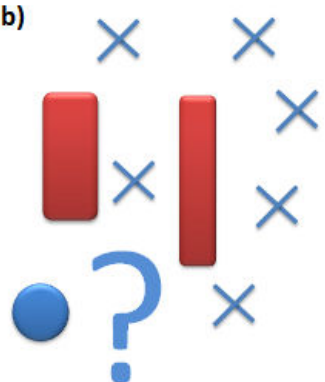

Fig. 11 - Planning towards: a) one target and b) multiple targets

This robot uses a very compact CPU and a small processing unit that cannot run expensive time consuming algorithms. Moreover, the planning algorithm must also take into account the kinematic constraints of the robot. A new fast and efficient motion planning algorithm is being developed. The foundations of the new algorithm are presented next.

\subsubsection{Motion Planning Algorithm}

A heuristic approach concerning the motion planning algorithm is being developed.

First of all let's clearly state the problem: Collect at least a given amount of balls on a golf practice field (Driving Range) while maximizing the efficiency $(\eta)$.

$$
\eta=\frac{\text { number of collected balls }}{\text { distance travelled }} .
$$

Restrictions may apply in the form of minimum number of balls to collect, minimum distance travelled or maximum time spent. A necessary condition is that the initial and final positions are the same.

It is assumed that golf balls location is given a priori.

Developing an efficient algorithm implies to be concerned about optimality. Computing optimal motion plans is a very challenging problem even in simple cases (J.Canny and J.H.Reif, 1987). Sampling based algorithms have proven to be a very intelligent choice in terms of computational expenditure although compromising the completeness (Karaman and Frazzoli, 2010, Latombe, 1991, Kavraki et al., 1996, LaValle, 2006). Recently Karaman and Frazolli (Karaman and Frazzoli, 2011) presented improved versions of two of the most known sampling based algorithms, PRM (Probabilistic Roadmaps) (Kavraki et al., 1996) and RRT (Rapidly Exploring Random Trees) (Kuffner and Lavalle, 2000). They presented asymptotically optimal incremental sampling algorithms, which means, that they approach the best solution as the number of iterations grows up. The RRT has the advantage over PRM that does not need a learning phase (LaValle, 2006). The RRT* has been reported to be very effective even when applied to robots with several DOF 
(Degrees of Freedom) (Karaman et al., 2011, Perez et al., 2011).

Our approach consists of having two trees growing from the same point, each one with the same characteristics of the RRT* algorithm. One is called the forward tree (Ta) and the other the backward tree (Tb) (Fig. 12), similarly with the RRT-connect algorithm (Kuffner and Lavalle, 2000), but in this case both have the same root $\left(x_{\text {init }}\right)$. Whenever a new sample is added to one tree, it tries to connect to the other tree closing a complete loop. The efficiency $(\eta)$ is improved whenever a better path is found. The new algorithm, called Twin-RRT*, is summarized as follows:

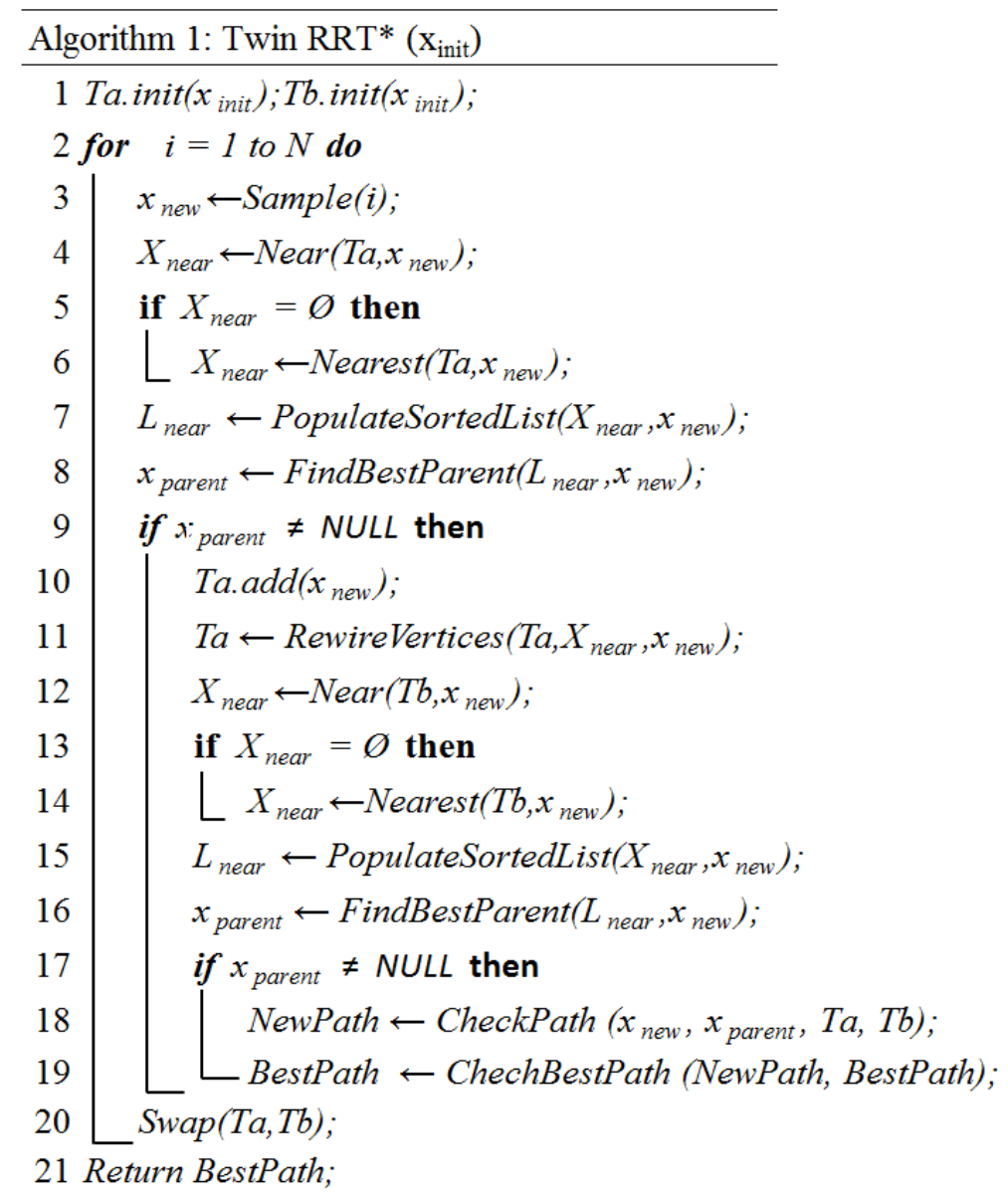

The PopulateSortedList, Near, RewireVertices and FindBestParent sub-routines may be found elsewhere (Perez et al., 2011). 


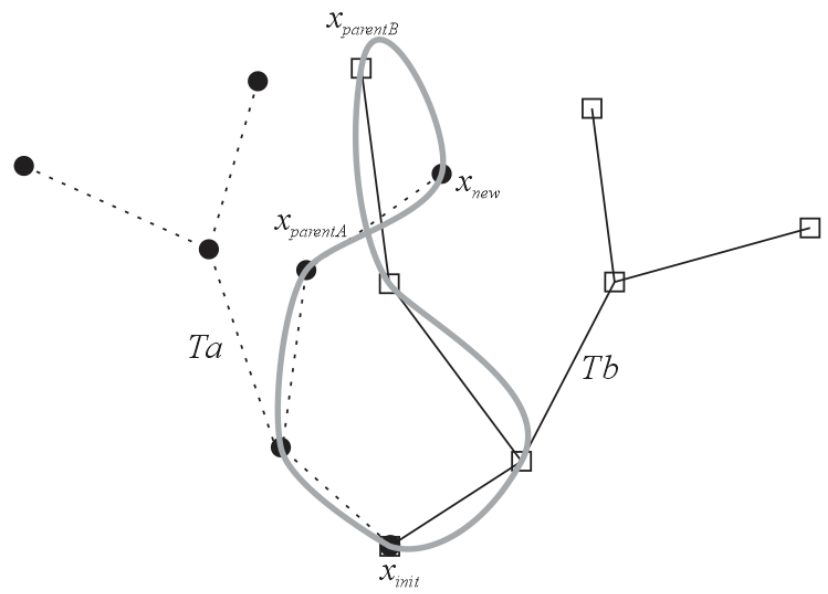

Fig. 12 - New Proposed Twin-RRT* Algorithm. The Forward (Ta) and the Backward (Tb) trees, both share the same root $\left(x_{\text {init }}\right)$. A complete path (curved loop) is formed whenever the two trees connect and the obtained path complies with the imposed restrictions.

The final generated path already takes into account the kinematic constraints, by using Dubins Curves [20]. It is assumed for simplicity that all curves are made at the maximum turning angle, so the curvature has radius $\rho_{\text {min }}$.

\section{RESULTS}

\subsection{Field Tests}

The prototype robot built was submitted to some practical tests performed on a real practice field (Fig. 13). It easily climbs a $22^{\circ}$ slope terrain. The maximum reached speed was $8 \mathrm{~km} / \mathrm{h}$, which is higher than the average buggy operation speed $(5 \mathrm{~km} / \mathrm{h})$. The full container capacity is over 1,200 golf balls and the maximum turning angle is $20^{\circ}$.

The average power consumption of the whole system is $1,600 \mathrm{~W}$. The first test log file revealed that the OMNI_3MD_MAX motor controller board maximum temperature registered was $65^{\circ} \mathrm{C}$ which is well below the cut-off temperature $\left(100^{\circ} \mathrm{C}\right)$. The robot takes $140 \mathrm{~min}$ to completely sweep a 25,000 $\mathrm{m} 2$ field at average speed of $7.2 \mathrm{~km} / \mathrm{h}(2 \mathrm{~m} / \mathrm{s})$. Considering a case in which 1,200 balls are evenly distributed over the field, it would collect them all in $140 \mathrm{~min}$. Considering a working time of $8 \mathrm{~h} /$ day the robot can travel approximately $57.6 \mathrm{~km}$ which represents covering 3.4 times the total area. 


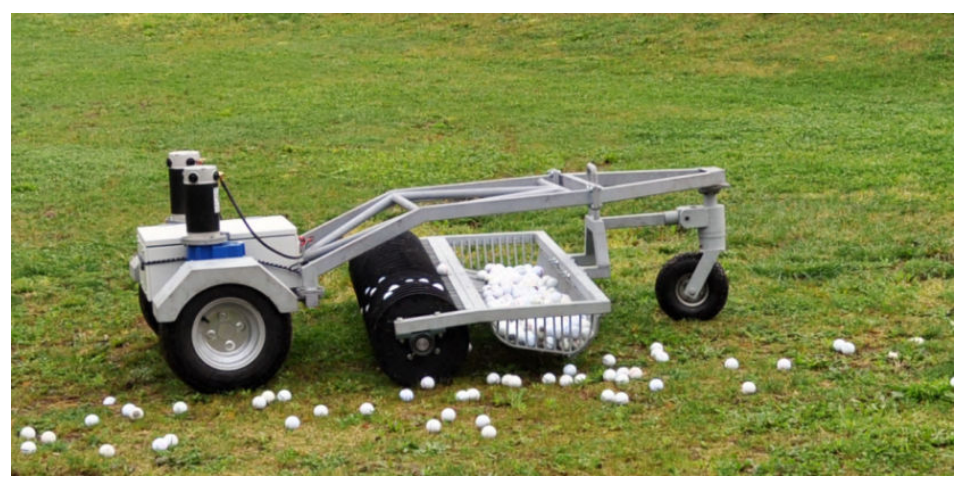

Fig. 13 - Golf Ball Picker Robot Prototype

\subsection{Path Planning Simulation Results}

A set of 100 simulations were performed with the new Twin RRT* algorithm, on a machine equipped with an Intel ${ }^{\circledR}$ Xeon ${ }^{\circledR}$ Processor X3430 (8M Cache, $\left.2.40 \mathrm{GHz}\right)$ and 8 GB RAM. The number of iterations was limited to 10,000 . Three degrees of freedom were considered for the robot configuration, namely the position $\mathrm{x}$ and $\mathrm{y}$ and the direction $\theta$. The $\mathrm{x}$ and $\mathrm{y}$ values are integers and the direction has been discretized by multiples of $10^{\circ}$. A field of $250 \mathrm{~m}$ length and $100 \mathrm{~m}$ width was considered for the simulation with 10,000 balls randomly distributed.

The algorithm is able to generate closed loop trajectories, retrieving a feasible path and maximizing efficiency $(\eta)$ over time (Fig. 14). From this chart, one can easily see that in just a few seconds an acceptable solution is reached, even though it continues searching for better results. It is possible to retrieve the best path found at any moment and start to navigate. The anytime flavor of the RRT* algorithm is an interesting feature for real-time applications. The Twin-RRT* continues to improve the path over time towards an optimal solution. It retrieves the first solution in just a few milliseconds and shows a rapid convergence to the optimal solution.

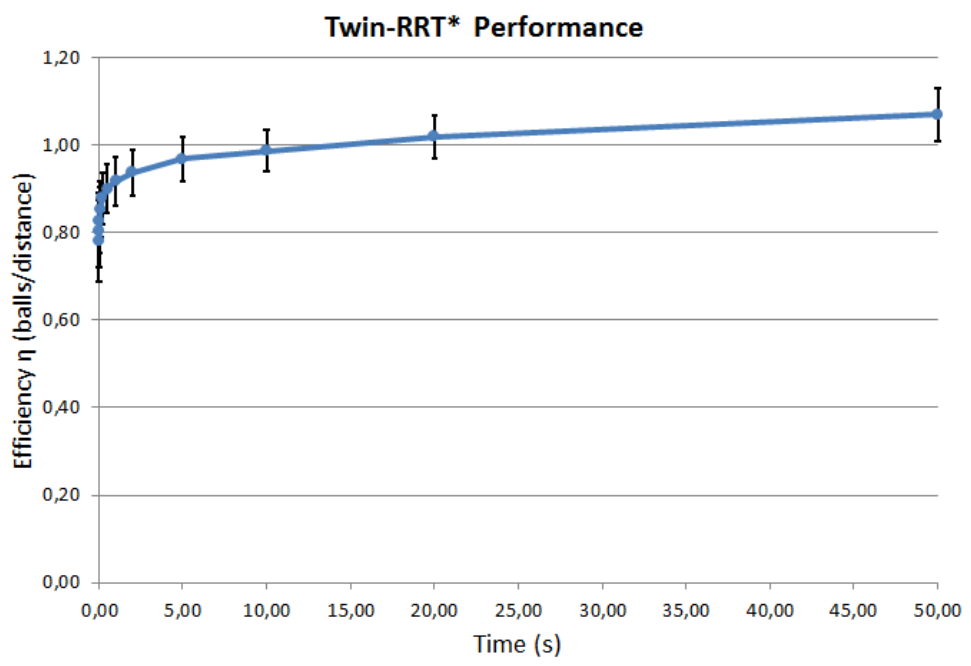

Fig. 14 - Twin RRT* Performance over time. 


\subsection{Patents}

There are patents pending ("Fully autonomous or remotely operated golf ball picking system" Portuguese Patent 103 807, and United States under assessment, Patent on 30 September 2010: US-2010-0250024-A1 and "SIMPLO - Hybrid Real-Virtual Electronic Information System" - Submitted on December 2011) describing the technologies employed in this project, which makes this an attractive product for industry. This project is being developed in consortium with SAR - Soluções de Automação e Robótica, University of Minho and Partis Consulting.

\section{CONCLUSIONS}

This paper describes the industrial design and implementation of an autonomous robot to pick up golf balls, following technical specifications. These were previously determined by a group of people, including golf players, electrical and mechanical engineers and golf association directors, amongst others.

The overall system architecture and component integration was presented and field tests were conducted to evaluate if technical specifications have been met. Preliminary results demonstrate that the prototype meets the technical requirements concerning motors and batteries dimensioning, mechanical robustness, capacity (1200 balls), speed $(2 \mathrm{~m} / \mathrm{s})$, maximum slope $\left(22^{\circ}\right)$ and maximum turning angle $\left(45^{\circ}\right)$.

This work is being developed jointly with industry, willing to manufacture and to export the final product. A hybrid information system has been developed to statistically predict the golf balls location on the field. A new path planning algorithm is being developed which shares similarities with both RRT-connect and RRT*. This new Twin-RRT* algorithm produces closed loop trajectories and complies with the robot kinematic constraints. It may be used to solve some TPP related problems while being computationally feasible.

\section{FURTHER WORK}

The autonomous navigation feature will soon be implemented on the real robot. The path planning strategy has shown preliminary promising results. Field tests are still required to prove the adequacy of the planner to real environments. The Twin-RRT* algorithm will be improved to suit higher DOF (Degrees of Freedom). Obstacle avoidance is also being introduced on the algorithm.

Regarding the final prototype, a lighter structure is already being designed (Fig. 15) and will soon be manufactured. 


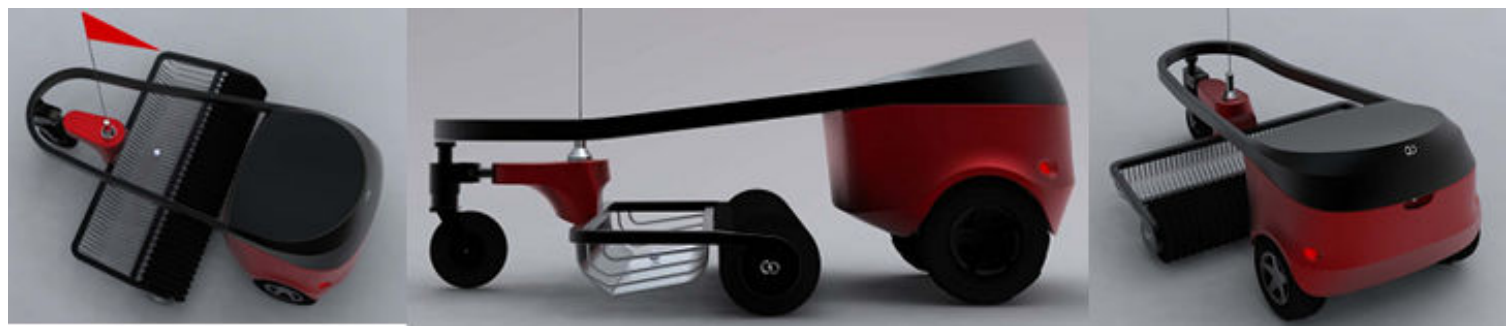

Fig. 15 - Final golf ball picker robot design

\section{ACKNOWLEDGEMENTS}

The authors would like to thank the Portuguese Science Foundation - FCT "Fundação para a Ciência e Tecnologia" for the PhD Grant No. SFRH / BD / 43008 / 2008, the Portuguese Golf Federation, and Mecanarte, Metalúrgica da Lagoa Lda for all the support.

The project GOLFmINHO n ${ }^{\circ} 1583$ is co-financed by European community fund FEDER (Fundo Europeu de Desenvolvimento Regional), approved by QREN (Quadro de Referência Estratégico Nacional, Portugal 2007.2013) and supported by ON.2 (o Novo Norte - Programa Operacional da Região Norte), CCDRn (Comissão de Coordenação e Desenvolvimento Regional do Norte) and ADI (Agência de Inovação). 


\section{REFERENCES:}

AUTOMATION, S. 2011. Golf Ball Picker Robot [Online]. Available: http://www.softeeautomation.com/html/ballpicker.htm [Accessed 2011-05-24 2011].

CHOSET, H. 2005. Principles of Robot Motion: Theory, Algorithms, and Implementation, MIT Press (C) 2005

DE LOOZE, M. P., STASSEN, A. R. A., MARKSLAG, A. M. T., BORST, M. J., WOONING, M. M. \& TOUSSAINT, H. M. 1995. Mechanical loading on the low back in three methods of refuse collecting. Ergonomics, 38, 1993 2006

DUDEK, G. \& JENKIN, M. 2010. Computational Principles of Mobile Robotics, Cambridge University Press.

GOLDEN, B. L., LEVY, L. \& DAHL, R. 1981. Two generalizations of the traveling salesman problem. . OMEGA, 9, 439-445.

J.CANNY \& J.H.REIF 1987. New lower bound techniques for robot motion planning problems. IEEE Symposium on Foundations of Computer Science (FoCS). Los Angeles,CA.

JINWEI, G., RAVI, R., PETER, B. \& SHREE, N. 2009. Removing image artifacts due to dirty camera lenses and thin occluders. ACM Trans. Graph., 28, 1-10

KARAMAN, S. \& FRAZZOLI, E. 2010. Incremental Sampling-based Algorithms for Optimal Motion Planning. CoRR, $\mathrm{abs} / 1005.0416$.

KARAMAN, S. \& FRAZZOLI, E. 2011. Sampling-based algorithms for optimal motion planning. Int. J. Rob. Res., 30, 846-894.

KARAMAN, S., WALTER, M. R., PEREZ, A., FRAZZOLI, E. \& TELLER, S. Anytime Motion Planning using the RRT*. Robotics and Automation (ICRA), 2011 IEEE International Conference on, 9-13 May 20112011. 1478-1483.

KAVRAKI, L. E., SVESTKA, P., LATOMBE, J. C. \& OVERMARS, M. H. 1996. Probabilistic roadmaps for path planning in high-dimensional configuration spaces. Robotics and Automation, IEEE Transactions on, 12, 566-580.

KUFFNER, J. \& LAVALLE, S. RRT-connect: An efficient approach to single-query path planning. Proc. IEEE Int. Conf. Robot. Autom. (ICRA, 2000. 995-1001.

LATOMBE, J. C. 1991. Robot Motion Planning, Boston, MA, Kluwer Academic Publishers.

LAVALLE, S. M. 2006. Planning algorithms, Cambridge University Press.

LI, Y. F. \& CHEN, S. Y. 2003. Automatic recalibration of an active structured light vision system. Robotics and Automation, IEEE Transactions on, 19, 259-268

LIMA, P., BONARINI, A., MACHADO, C., MARCHESE, F. M., MARQUES, C., RIBEIRO, F. \& SORRENTI, D. G. 2001. Omni-Directional Catadioptric Vision for Soccer Robots. Special Issue of the Robotics and Autonomous Systems Journal, Elsevier, Amsterdam, 36, 31.

MACHADO, C., CAMPOS, F., MARTINS, M., SAMPAIO, S. \& RIBEIRO, F. 1999a. Footballer Autonomous Mobile Robot - Control and Vision System. Festival International de Science et Technologie. Bourges, France.

MACHADO, C., CAMPOS, F., MARTINS, M., SAMPAIO, S. \& RIBEIRO, F. 1999b. Mechanics and Electronics of the Footballer Autonomous Mobile Robot. Festival International de Science et Technologie. Bourges, France. 
MING-SHYAN WANG, C.-C. W. 2006. A Mobile Robot for Ball Picking. Proceedings of 2006 CACS Automatic Control Conference, St. John's University, Tamsui, Taiwan.

ONG, H. L. 1982. Approximate algorithms for the traveling purchaser problem. Operations Research Letters, 1, 201-205.

PACHECO, L. F. C., OLIVEIRA, ANDRÉ J.B.DE, RIBEIRO, A.F. 2008. Mobile robot for autonomous golf balls picking. Controlo 2008 : proceedings of the Portuguese Conference on Automatic Control. Vila Real, Portugal.

PACK, R. T., WILKES, D. M. \& KAWAMURA, G. A software architecture for integrated service robot development. Systems, Man, and Cybernetics, 1997. Computational Cybernetics and Simulation., 1997 IEEE International Conference on, 12-15 Oct 1997 1997. 3774-3779 vol.4.

PEARN, W. L. \& CHIEN, R. C. 1998. Improved solutions for the traveling purchaser problem. Computers \&amp; Operations Research, 25, 879-885.

PEREZ, A., KARAMAN, S., WALTER, M., SHKOLNIK, A., FRAZZOLI, E. \& TELlER, S. 2011. Asymptotically-optimal Manipulation Planning using Incremental Sampling-based Algorithms. IEEE/RSJ International Conference on Intelligent Robots and Systems (IROS).

RAMESH, T. 1981. Traveling purchaser problem. OPSEARCH, 18, 78-91.

RAVI, R. \& SALMAN, F. 1999. Approximation Algorithms for the Traveling Purchaser Problem and Its Variants in Network Design

Algorithms - ESA' 99. In: NEŠETRIL, J. (ed.). Springer Berlin / Heidelberg.

RIBEIRO, F. 2007a. ENIGMA - Cadeira de Rodas Omnidireccional. Journal Robótica, 66, 50-51.

RIBEIRO, F., MOUTINHO, I., SILVA, P., FRAGA, C. \& PEREIRA, N. 2004. Controlling Omni-directional Wheels of a MSL RoboCup Autonomous Mobile Robot. Robotica'2004. Porto.

RIBEIRO, F., MOUTINHO, I., SILVA, P., BRAGA, P. AND PEREIRA, N. 2007b. Mobile Robot Construction for Edutainment Application. Revista Robótica, 69, 12-16

SINGH, K. N. \& VAN OUDHEUSDEN, D. L. 1997. A branch and bound algorithm for the traveling purchaser problem. European Journal of Operational Research, 97, 571-579.

SUN-LI, W., MING-YANG, C. \& WEN-CHUNG, H. 2005. Design and implementation of a prototype vision-guided golf-ball collecting mobile robot. Mechatronics. 2005. ICM'05. IEEE International Conference on.

ULRICH, K. T. \& EPPINGER, S. D. 2004. Product Design and Development, McGraw-Hill, New York.

WADA, K. 2009. Tutorial 1: Theoretical Aspects of Autonomous Mobile Robots. Parallel and Distributed Computing, Applications and Technologies, 2009 International Conference on.

YANG, J., NAI GUANG, L. \& DONG, M. Computer Vision Calibration New Easy Method. Information Technology and Computer Science, 2009. ITCS 2009. International Conference on, 25-26 July 2009 2009. 102-105 